\title{
Regarding Lui K.J. (2006). Interval estimation of risk difference in simple compliance randomized trials. JMASM, 5, 395-407.
}

Ian R. White

MRC BioStatistics Unit

Follow this and additional works at: http://digitalcommons.wayne.edu/jmasm

Part of the Applied Statistics Commons, Social and Behavioral Sciences Commons, and the Statistical Theory Commons

\section{Recommended Citation}

White, Ian R. (2007) "Regarding Lui K. J. (2006). Interval estimation of risk difference in simple compliance randomized trials. JMASM, 5, 395-407.," Journal of Modern Applied Statistical Methods: Vol. 6 : Iss. 2 , Article 31.

DOI: $10.22237 /$ jmasm/1193891400

Available at: http://digitalcommons.wayne.edu/jmasm/vol6/iss2/31 


\section{Letters to the Editor}

Lui K. J. (2006). Interval estimation of risk difference in simple compliance randomized trials. Journal of Modern Applied Statistical Methods, 5, 395-407.

Ian R. White

MRC Biostatistics Unit

Professor Lui (2006) reports a careful comparison of the properties of six possible interval estimators for the causal risk difference among treatment-compliers ${ }^{1}$. He recommends for general use the confidence interval based on a $\tanh ^{-1}$ transformation of the causal risk difference, on the grounds that it has at least the nominal coverage and it has the smallest mean length of all the methods.

However, the second of these criteria is not self-evidently the most relevant, and there are other possible criteria which would point to a different choice of interval estimator.

1. Some interval estimators with large mean length are valuable and in common use. An example is the number needed to treat, defined as the inverse of the risk difference. The appropriate confidence interval for the number needed to treat includes the inverse of all values in the confidence interval for the risk difference: in particular, it includes infinity if the confidence interval for the risk difference includes zero ${ }^{2}$. This interval in fact has infinite mean length, but it remains appropriate and widely used, if sometimes misunderstood.

2. More generally, mean confidence interval length is a scale-dependent criterion: when the parameter is transformed to a different scale, confidence intervals retain their coverage properties but not their mean length. Thus mean length on different scales could have been considered.

3. Rather than require coverage to be at least the nominal coverage, one could require coverage that is close to the nominal coverage. Professor Lui's recommended method has over $98 \%$ coverage for nominal $95 \%$ confidence intervals in many of the simulation settings.
4. A further criterion in the treatmentcompliance setting is that one could require confidence intervals to agree with the intention-to-treat $\mathrm{P}$-value, by excluding zero if and only if the intention-to-treat test is significant. This is an appropriate requirement because the null hypotheses for the intention-to-treat and complianceadjusted analyses are the same and there is no gain in power from allowing for noncompliance in this setting ${ }^{3}$. Confusion in interpretation could easily arise if adjustment for non-compliance in a particular data set appeared to change a nonsignificant result into a significant one or vice versa.

The Fieller's theorem confidence interval has properties 3 and 4 above ${ }^{4}$. By its derivation, it agrees exactly with the intention-to-treat $\mathrm{P}$-value computed from an asymptotic test (use of an exact intention-to-treat test would make the equivalence only approximate). Its coverage is therefore close to the nominal, as shown in Professor Lui's simulation study. I therefore believe that the Fieller's theorem confidence interval should also be considered for use in practice, especially when testing the null hypothesis of no intervention effect is important.

\section{References}

Altman DG. Confidence intervals for the number needed to treat. British Medical Journal 1998; 317: 1309-1312.

Branson M, Whitehead J. A score test for binary data with patient non-compliance. Statistics in Medicine 2003; 22: 3115-3132.

Frost C, Thompson SG. Correcting for regression dilution bias: comparison of methods for a single predictor variable. Journal of the Royal Statistical Society (A) 2000; 163: 173189. 